\title{
Téoros
}

Revue de recherche en tourisme

\section{Le patrimoine est-il soluble dans le tourisme ?}

\section{Luc Noppen et Lucie K. Morisset}

Volume 22, numéro 3, automne 2003

URI : https://id.erudit.org/iderudit/1071350ar

DOI : https://doi.org/10.7202/1071350ar

Aller au sommaire du numéro

Éditeur(s)

Université du Québec à Montréal

ISSN

0712-8657 (imprimé)

1923-2705 (numérique)

Découvrir la revue

Citer cette note

Noppen, L. \& Morisset, L. K. (2003). Le patrimoine est-il soluble dans le tourisme? Téoros, 22(3), 57-59. https://doi.org/10.7202/1071350ar d'utilisation que vous pouvez consulter en ligne.

https://apropos.erudit.org/fr/usagers/politique-dutilisation/ 


\author{
Chaire de recherche du Canada en patrimoine urbain
}

\title{
Le patrimoine est-il soluble dans le tourisme?
}

\section{Luc Noppen et Lucie K. Morisset}

D epuis Lascaux ou Macchu Picchu, l'essor de l'écotourisme et du développement durable ont remis à l'honneur l'idée voulant que le tourisme soit néfaste pour le patrimoine. Marchandage au temple ou simple envahissement (matériel ou idéologique), l'ICOMOS ${ }^{1}$ est d'ailleurs formel à cet égard : la question « comment faire en sorte que le tourisme ne constitue pas un danger pour le patrimoine ? » figure au préambule des interrogations de l'UNESCO sur « l'authenticité $»^{2}$.

L'histoire nous apprend pourtant le contraire ou, à tout le moins, l'inextricable relation entre le tourisme et le patrimoine. Celui-ci, comme phénomène et comme objet de représentation, s'ancre en effet au regard de «l'Autre »... dont le touriste, mépris pour un parasite, a traditionnellement endossé le rôle. Mais les rapports entre le tourisme et le patrimoine ont bien changé. L'attitude positiviste de l'exploitation touristique contemporaine pourrait être en cause : en assumant que le patrimoine est un donné, un état objectif, on conclut que la mise en tourisme ne serait qu'une bonification économique résultant de la valorisation d'un site, d'une région ou d'un quartier. Les « rétrospectives du patrimoine » qui se sont multipliées dans les milieux scientifiques ces vingt dernières années ont plutôt révélé des constructions de l'esprit : le patrimoine est né de fabrications intellectuelles dont les mécanismes et les finalités ont évolué, de la même manière que les actions de mise en tourisme, avec les transformations sociales et la succession des perceptions du temps.

Admettons ainsi d'emblée qu'aucun patrimoine n'existe ou n'a existé « en soi ». La valorisation des monuments historiques, tout comme celle, subséquente, des « centres historiques », est apparue à la fin du XVIII' siècle, quand l'Occident a adhéré à un renouveau de la pensée qui instituait l'expérience du monde comme seule productrice de savoirs. Opposée au rationalisme, cette doctrine «empiriste », comme on l'a depuis nommée, postulait que l'observation des objets et des phénomènes devait remplacer, par exemple, les spéculations philosophiques au titre de processus scientifique, en vue d'assurer le progrès de l'humanité. Aux côtés des sciences naturelles qui prirent ainsi leur envol, l'archéologie et l'histoire de l'art se sont dès lors évertuées à caractériser et à classer les productions de l'humanité dont on constatait la diversité, tout en remarquant, pour la première fois, l'implacable déroulement du temps. Imposé par la notion même de progrès qui supposait la succession linéaire « d'époques » différentes, un écart nouveau entre le présent et le passé consacrait, du coup, des objets rares et précieux, révélateurs de temps révolus. Cette sacralisation des «monuments ${ }^{3}$ » - ceux de l'Antiquité d'abord, ceux du Moyen-Âge ensuite impliquait cependant un témoin, un « Autre », dont le regard, puis l'intérêt, garantirait la préciosité. Comme l'anthropologie le soulignait à ce moment-là, le regard local, faute de distance et, surtout, de référence, manquait de compétence dans l'évaluation de la spécificité ou de l'originalité : intervint alors le bien connu Tour-ist (Marc Boyer ${ }^{4}$ en a, depuis, abondamment documenté l'histoire) qui, avec son regard comparatif et (donc) connaisseur, engendra ainsi les premières consécrations patrimoniales.

En créant de la sorte le patrimoine qu'il cautionnait, le tourisme ne servait évidemment pas que la science et la soif de connaissances qui, d'un pays à l'autre, ont embrassé le collectionnement des "antiquités». Le catalogue actuel des hauts lieux mondiaux du tourisme démontre que ses objets et ses sites patrimoniaux sont tous nés en appui aux constructions nationales, typiques du $\mathrm{XIX}^{\mathrm{c}}$ siècle européen qui, au $\mathrm{XX}^{\mathrm{c}}$ siècle, en a diffusé le modèle de par le monde. La fréquentation des temples grecs, des pyramides d'Égypte, des cathédrales françaises, tout comme la consommation de vins de Bordeaux et de porto, de fromage parmesan, bien avant l'avènement du tourisme de masse qui mettrait en lumière leurs avantages économiques, ont attiré l'attention des États sur le potentiel identitaire du «patrimoine » et sur son rôle dans l'édification des Nations. Ainsi dotés d'une finalité, les «monuments nationaux » ont constitué le patrimoine d'alors : c'est encore celui qui domine les itinéraires touristiques puisque, avec l'identité nationale, l'idéal de spécificité qu'il incarnait est demeuré, des pubs anglais au chalet suisse, en passant par la maison québécoise.

Mais alors que ce premier patrimoine a été consacré par les Dilettanti et autres personnels plus ou moins au service de la cause politique, le succès de l'institution patrimoniale a stimulé la formation d'une expertise dédiée, sorte de professionnalisation 
de « l'Autre ». Archéologues, historiens d'art et d'architecture, conservateurs de musée ont très tôt développé un nouveau champ d'intervention : celui des monuments historiques qu'il leur revenait de caractériser, de conserver et de mettre en valeur. Or, au $\mathrm{XX}^{\mathrm{e}}$ siècle, l'avènement de la nouvelle objectivité et des avantgardes a convenu de l'autonomie et de l'indépendance de ces spécialistes ; en s'appropriant le précepte libéral du travail voué au bien-être des populations, l'époque sous-tendait en effet que les experts seuls savaient les besoins et les solutions du «peuple »- justifiant d'une même venue la bureaucratie et lesdites « dictatures »du prolétariat. Déjà plus que centenaire, la symbiose entre le tourisme et le patrimoine commençait à s'effriter.

La seconde «vague » de consécrations patrimoniales s'est alors faite au nom d'une scientificité autoproclamée avec, à la clé, moins de succès populaire et d'impact touristique. Les objets ou les sites ont souvent été sélectionnés en fonction de critères techniques ou typologiques, par exemple, c'est-à-dire complexes, voire abscons et, surtout, intrinsèques aux disciplines auxquelles se référait leur identification. Ce choix dévolu à ne satisfaire qu'un public (très) averti, s'il a parfois eu pour avantage d'actionner le potentiel de reconnaissance et, partant, la conservation de patrimoines obscurs, avait un inconvénient : celui de dévaloriser le regard de l'Autre et, du même coup, la contribution du tourisme à la naissance patrimoniale, d'autant que le tourisme de masse qui déferlait alors appelait un jugement sans appel des « experts ». L'affection populaire dont on en venait à garder le patrimoine ne pouvait en effet que dégrader « l'authenticité » savamment construite par l'avantgarde, seul public véritablement en mesure d'apprécier les hauts lieux sanctifiés. En conséquence, un nombre important de «patrimoines » protégés n'ont jamais été mis en valeur ou même visités. Le catalogue québécois des monuments et des sites classés compte une majorité d'exemples ainsi parfaitement inconnus du grand public, de patrimoines qui sont peu ou pas utilisés dans le récit identitaire national, voir même régional ou local.

À la fin des années 1960, le tourisme n'avait pourtant pas encore épuisé ses ressources au secours du patrimoine. Mais une troisième vague de consécrations patrimoniales survint. Dans la foulée des mai 1968 et autres Berkeley, le recyclage des avant-gardistes - convertis du marxisme-léninisme en animateurs de luttes sociales - a fait du patrimoine l'outil de contre-attaque des grands projets d'un Urban Renewal déjà déclinant. Les figures familières d'un paysage construit jaugé à l'aune de la proximité ont été l'une après l'autre adoptées comme emblèmes d'un urbanisme de résistance, force d'opposition à l'autorité et processus d'empowerment. Monuments et sites protégés importaient souvent moins que l'action de contestation : les constructions patrimoniales sont alors devenues des écoles de démocratie populaire, passages obligés de plusieurs politiciens en herbe qui, tout de même, ont légué plusieurs acquis, à commencer par les plages importantes de ce patrimoine urbain préservées par des coopératives d'habitation, des ensembles de logement social ou des équipements collectifs qui animent toujours nos villes. Mais, tout en embrigadant une expertise qui, depuis, doit prendre position dans

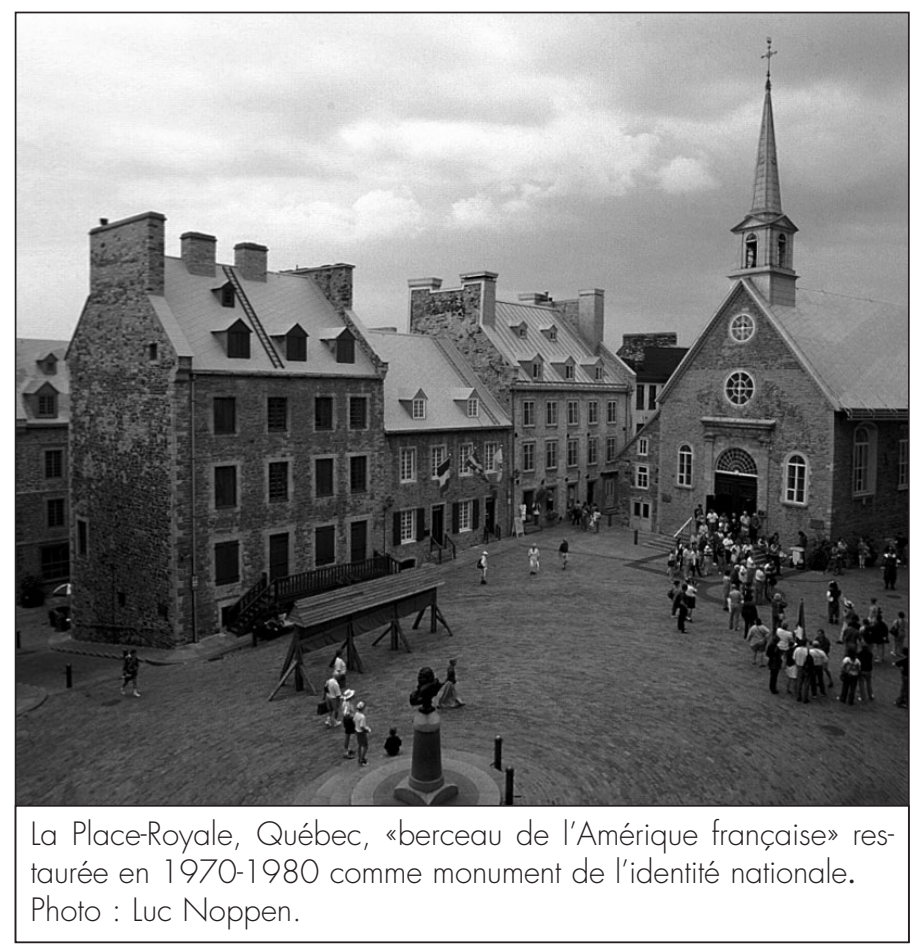

l'opposition diamétrale du projet de développement (mauvais dans tous les cas) à « l'héritage » (quel qu'il soit), ce qui a pu paraître à certains comme une démocratisation du patrimoine a engendré, d'une part, le rejet de la valeur d'art - mais il pourrait ne s'agir là que d'un constat « d'expert »-, d'autre part, et surtout, un mépris féroce du tourisme et de la valorisation touristique, jugés incompatibles avec la qualité de vie citoyenne.

Cette attitude devait conduire à la banalisation d'un patrimoine de plus en plus local, ingrédient parmi d'autres de la planification urbaine : aujourd'hui, la préparation des plans d'urbanisme et des schémas d'aménagement est le lieu de caractérisation du patrimoine et de limitation des projets urbains qui le menaceraient. Il s'agit d'un « patrimoine de proximité », produit par une collectivité désireuse de préserver son environnement et à qui la somme des protections réglementaires assure la stabilité recherchée. Ce «patrimoine de propriétaires » - bien que l'idée soit antinomique du concept patrimonial - concerne ainsi surtout les secteurs privilégiés par les résidants - à Montréal, le « plateau », le village gai, le boulevard Saint-Laurent, le quartier chinois sont des exemples -, tandis qu'on tend plutôt à contenir le tourisme dans les sites abandonnés par les habitants, ou en voie de l'être (le Vieux-Port de Montréal, le Vieux-Québec), dans un nouveau mouvement de ségrégation fonctionnelle de l'espace urbain (Cité du commerce international, Cité du multimédia, Quartier des spectacles, etc.).

Pendant que le monument se dissout ainsi dans le patrimoine, le regard de l'Autre est dorénavant condamné pour ingérence : sous le coup de la dénonciation rétrospective desdits « pillages » d'Italie, de Grèce ou d'Égypte aux XVIII ${ }^{\mathrm{e}}$ et $\mathrm{XIX}^{\mathrm{e}}$ siècles, le touriste fait figure d'intrus dans l'autonomie locale. Apparaissent alors de curieuses rémanences : au Québec, où le regard de l'Autre, depuis la fin du 
$\mathrm{XIX}^{\mathrm{e}}$ siècle, était celui de l'Étasunien, toute discussion sur l'avenir de quelque œuvre d'art, bien mobilier ou architecture historique semble ainsi vouée à une conclusion devenue caricaturale, celle voulant que nos «trésors nationaux » soient pillés par des « riches collectionneurs américains très portés sur les 'Canadiana' » ou des «Américains [qui] ont voulu [nous] acheter ${ }^{5}$.

Les conséquences de la rupture entre le tourisme et le patrimoine sont néanmoins plus concrètes et peut-être plus graves. Rupture, en effet, car on conçoit bien que, depuis la troisième vague de consécrations patrimoniales, les exploitants touristiques entretiennent une certaine circonspection à l'égard du patrimoine et préfèrent fuir tout objet potentiel de contestation organisée. Cela d'autant que le «patrimoine de proximité » se révèle peu satisfaisant pour un tourisme, devenu industrie entre-temps, toujours en quête de dépaysement, mais aussi de quelque spectacle : le patrimoine - pourtant haut lieu de la spécificité et, partant, d'un dépaysement garanti - se disqualifiant à ce titre, l'imagineering arrive en renfort de l'offre, produisant « seigneuries », « circuits de fantômes » et autres « villages » fantastiques, le plus loin possible des villes ou le moins en lien, dans tous les cas, avec le tissu urbain.

Cette « fuite en avant» bien justifiable achoppe pourtant à deux écueils. Le premier concerne le tourisme et a priori le tourisme urbain. Dans la mesure où l'expression identitaire des sites est évacuée en même temps que le patrimoine, cet « objet » né du XVIII ${ }^{e}$ siècle dont les efforts de caractérisation ont inventé « l'ailleurs », le dépaysement et le déplacement, Montréal - Club Med pour Club Med - n'est ni plus ni moins attirante ou typique que New York, Paris ou Boston. De fait, dans la ville de la mondialisation, les quartiers chinois, grecs, italiens ou gais, s'ils satisfont le Montréalais qui y représente sa modernité et sa mixité, d'une part se réfèrent à une polysémie du local relativement absconse pour un touriste " ordinaire », d'autre part dépayseront peu les Chinois, les Grecs, les Italiens ou les gais qu'on pourrait espérer y attirer. À l'extérieur des villes, l'imagineering de l'offre touristique met en lumière une semblable indifférence face à l'Autre : l'intérêt au Québec pour les anciens plateaux de tournage (par exemple de Robe Noire, devenu « site de la Nouvelle-France »- « Le seul site qui offre un paysage ressemblant aux descriptions de la Nouvelle-France ${ }^{6} »-$, de Marguerite Volant, aujourd'hui seigneurie de la Nouvelle-France) se dédie, à l'évidence, à un imaginaire franchement local, puisqu'il mise d'abord sur la notoriété créée, à cette échelle, par le film ou, plus encore, par la télésérie. L'Autre devient Nous.

Sans compter les effets économiques de cette touristification intérieure, le phénomène de représentation de soi qui affleure ici révèle le second écueil, voué celui-là au patrimoine, voire au sens que nous voulons donner à notre avenir collectif. Latente dans l'invention du "patrimoine de proximité », la forclusion du regard de l'Autre emprisonne le patrimoine dans un passé dont nous nous détachons davantage chaque jour. S'il est vrai que l'instrumentation patrimoniale des constructions nationales de jadis peut inciter à la méfiance, nul besoin de « jeter le bébé avec l'eau du bain » : l'exemple d'une France maintenant française, qui donc n'a plus besoin de monuments français, ou celui d'un Québec qui serait maintenant québécois démontre que le patrimoine doit être le fait de constantes réinventions, afin, perpétuellement, de correspondre aux valeurs et aux sensibilités des nouveaux temps. Or, le tourisme était un ingrédient actif de cette continuelle re-construction de la linéarité historique, en consacrant, comme nous l'avons exposé, les témoins d'époques successivement poussées vers le passé par l'avancement du progrès. En dissociant le tourisme du patrimoine et, ce faisant, en excluant l'Autre de notre identité, on confine le patrimoine (cette "vieille chose ») au passéisme et le tourisme à l'approximation des coureurs des bois, des patriotes et autres « Nouvelles-Frances » en vogue, toutes figures, comme l'a montré Hartog (2003), « chargées d'une dette tant à l'égard du passé que $d u$ futur $»$. Le plateau, le village gai ou le boulevard Saint-Laurent sont les fantômes de cet univers captif du présent - du nôtre.

Quant au tourisme, il s'accroche pourtant encore, parfois, à la valorisation de la spécificité. Mais privée d'un patrimoine agonisant, et que ses exégèses rendent au demeurant suspect, l'identité mise en tourisme ne se conjugue plus, elle aussi, qu'au présent : l'usage seul paraît dorénavant garant de cette « authenticité » prisée, comme en témoignent les écomusées plus nombreux chaque jour. Mais cela - l'abandon de l'objet pour l'usage ou le confinement du passé dans le présent - est une autre histoire. Tout comme l'est l'avenir de tous ces patrimoines que nous laissons derrière nous.

À l'évidence, le patrimoine n'est plus soluble dans le tourisme. Mais est-ce vraiment un gain?

Luc Noppen, titulaire de la Chaire de recherche du Canada en patrimoine urbain, et Lucie K. Morisset sont tous deux historiens d'architecture et professeurs au Département d'études urbaines et touristiques à l'École des sciences de la gestion de l'UQAM.

\section{Notes}

1 International Council of Monuments and Sites.

2 Voir, par exemple, le Forum UNESCO - Université et patrimoine, tenu à Beyrouth et Byblos le 12 décembre 2000.

3 Le « monument» que l'étymologie veut comme simple porteur de mémoire, a alors pris sa connotation d'exception (on dira d'une œuvre, par exemple, qu'elle est un « monument » en raison de son caractère remarquable), du fait justement, de l'échantillonnage du temps.

4 Lire entre autres Boyer (1996).

5 Voir, par exemple, Lemieux (2002).

6 Comme l'affirme la promotion touristique du site.

\section{Bibliographie}

Boyer, Marc, (1996), L'invention du tourisme, Paris, Gallimard.

Hartog, François, (2003), Des régimes d'historicité. Présentisme et expériences du temps, Paris, Seuil.

Lemieux, Louis-Guy, (2002), «La maison F.-X-Garneau est sauvée », Le Soleil, 12 février, p. B-1. 